\title{
Sesión de posters apoyada en plataformas de e-learning
}

\section{Luis Antonio Tortajada Genaro ${ }^{\text {a,b }}$, Rosa Puchades ${ }^{a}$}

a Departamento de Química. UNIVERSITAT POLITÈCNICA DE VALÈNCIA. Camí de Vera S/N, Valencia (España). 46022.

b Grupo de innovación docente TECOF “Trabajo y Evaluación de Asignaturas mediante Técnicas de Evaluación Continua Formativa”. UNIVERSITAT POLITÈCNICA DE VALÈNCIA. Camí de Vera S/N, Valencia (España). 46022.

\begin{abstract}
The poster sessions are a teaching-learning strategy, that simulates working scenarios in the university classroom. The success of this exercice is related to how the previous tasks have been addressed. In this communication, the results of a teaching action focused on the elaboration and exhibition of a poster as materialization of a learning process by inquiry are presented. Given its complexity, the proposed approach has been a colaborative activity and supported by a virtual educational space that facilitates an active and flexible interaction between teachers and students and between the students themselves. Furthermore, a system of continuous formative assessment has been set up so that the feedback can be highlighted during its development. The student results achieved show the excellent combination of quality and satisfaction for the academic work carried out. In addition to inducing the acquisition of knowledge in specialized topics, it has been possible to promote skills related to communication, critical thinking, creativity, and cooperation.
\end{abstract}

Keywords: inquiry-based learning, collaborative learning, poster evaluation, Sakai platform

\footnotetext{
Resumen

Las sesiones de posters son una estrategia de enseñanza-aprendizaje que permiten simular escenarios laborales en el aula universitaria. El éxito de esta actividad está relacionado con el modo con que se han abordado tareas anteriores. En esta comunicación, se presentan los resultados de una acción docente dirigida a la elaboración y exposición de un póster como materialización de un proceso de aprendizaje por indagación. Dada su complejidad, el enfoque propuesto ha consistido en una actividad colaborativa apoyada por un espacio virtual educativo que facilite una interacción activa y flexible entre los profesores y los alumnos, y entre los propios alumnos. Al mismo tiempo, se ha instaurado un sistema de evaluación continua formativa para que la retro-alimentación fuera una acción destacada durante su desarrollo. Los resultados alcanzados por los alumnos ponen de manifiesto la excelente combinación de calidad y satisfacción por el trabajo académico realizado. Además de inducir la adquisición de conocimiento en temáticas especializadas, se ha logrado fomentar habilidades relacionadas con la
} 
comunicación, pensamiento crítico, creatividad, trabajo en equipo y cooperación.

Palabras clave: aprendizaje por indagación, aprendizaje colaborativo, evaluación de posters, plataforma Sakai

\section{Introducción}

En un modelo educativo basado en la adquisición de competencias, el profesor debe inducir que el estudiante abandone una posición de pasividad en las aulas y buscar vías para cubrir sus necesidades particulares. Una interesante aproximación es el aprendizaje por indagación, o inquiry-based learning en inglés (Edelson et al. 1999). Se trata de una enseñanza reflexiva donde los alumnos deben construir el nuevo conocimiento, pero no se les proporciona toda la información necesaria para el desarrollo de las actividades, por lo que deben involucrarse en el problema plateado y aportar soluciones (Gormally et al. 2009). Este enfoque facilita la participación activa, ayuda a desarrollar el pensamiento crítico y fomenta la capacidad para resolver proyectos.

Realizar este aprendizaje por indagación de forma colaborativa es un área creciente de investigación pedagógica (Kaartinen y Kumpulainen, 2002; Wenzel, 2007; Redondo, 2008). Las habilidades más fuertes de un estudiante pueden compensar las habilidades más débiles de otro. Para alcanzar este objetivo, la principal limitación es encontrar maneras de apoyar adecuadamente la comunicación y el proceso de creación de conocimiento. En este punto, el papel del profesor es ayudar a proporcionar pautas y vías claras de colaboración.

Durante los últimos 20 años, el uso de las tecnologías de la información y comunicación han facilitado ese aprendizaje por descubrimiento. Actualmente, no se realiza la búsqueda de información sin utilizar buscadores web y bases de datos on-line. Dichas tecnologías también se han utilizado para crear ambientes que involucran a los estudiantes en actividades como simulaciones, visualización de datos, comunicación científica, etc. (Van Joolingen et al. 2007; Carabantes, 2010). Hay que destacar que los espacios digitales han pasado de ser un mero repositorio de contenidos y recursos, a convertirse en espacios en los que el estudiante puede llevar a cabo procesos de autoaprendizaje personalizados y colaborativos. Esa es la finalidad de las plataformas empleadas en la educación a distancia o e-learning (Yang y Tsai, 2010; Roffe, 2002). Proporcionan entornos holísticos para la prestación y gestión de experiencias educativas y poseen herramientas que apoyan la creación de cursos en línea, permiten compartir recursos, abren vías flexibles de corrección y retro-alimentación, y permiten gestionar tareas o exámenes. Destacan especialmente las plataformas denominadas Iniciativas de Código Abierto (Open source initiatives) tipo Moodle (www.moodle.org), Sakai (www.sakaiproject.org) y ATutor (www.atutor.ca), sistemas que permiten adaptarse a las necesidades particulares.

Si el aprendizaje por indagación concluye con la realización de un póster, el ejercicio posee un valor añadido (Sisak, 1997). Los posters son importantes para la distribución de información desde la investigación básica a la divulgación empresarial. En la futura carrera profesional, los egresados universitarios participarán en congresos, seminarios y ferias donde

(cc)) EY-NC-ND 2017, Universitat Politècnica de València 
presentar sus resultados o productos y observarán los de otros en formato de póster. Sin embargo, los estudiantes no conocen las características específicas que deben tenerse en cuenta en su preparación y su defensa (Newbrey, 2006). Por ello, varios investigadores han propuesto el póster para diferentes actividades académicas, desde la presentación de resultados de proyectos de investigación de pregrado hasta exponer los resultados de actividades de laboratorio (Wimpfheimer, 2004).

Diferentes autores han publicado el amplio rango de beneficios derivados de la realización de posters en el aula. Las sesiones de posters se centran en los estudiantes y fomentan un aprendizaje más profundo (Marino et al. 2000; Scott, 2008). Los animan a reflexionar sobre su aprendizaje durante el proceso, les obliga a enfatizar los importante frente a lo superficial, y les permiten demostrar su aprendizaje y aprender de las experiencias de otros estudiantes (Denzine, 1999). Este tipo de actividad fomenta la creatividad y los aspectos visuales de la temática estudiada y tienen la oportunidad de mejorar las habilidades de comunicación escrita, visual y verbal. Frente las presentaciones orales estándar, son consideradas menos intimidantes, lo que activa de forma más natural un flujo de discusión alrededor de las experiencias de los estudiantes (Stegemann y Sutton-Brady, 2009). Finalmente, la evaluación es más directa y sencilla, permitiendo diferentes aproximaciones respecto a los sistemas evaluadores (propio alumno, compañeros, profesor, externo o modelos mixtos). No obstante, el análisis de la bibliografía indica la necesidad de seguir investigando para que se afiance como una herramienta efectiva de enseñanza-aprendizaje.

\section{Objetivos}

El objetivo del estudio era la realización de una actividad basada en el aprendizaje por indagación colaborativo y cuyo resultado final se expusiera en una sesión de posters. Nuestra investigación se ha centrado en las herramientas que ayuden a que los estudiantes comprendan la meta marcada y el modo de alcanzarla, implicándolos en todo el proceso. Por tanto, se ha estudiado cómo planificar, desarrollar y evaluar la actividad, asistido por la herramienta de e-learning PoliformaT (tecnología Sakai). Para ello, se plantean objetivos secundarios

- Elaboración de materiales que transformen el reto académico ofrecido por los docentes en acción

- Establecimiento de vías de retro-alimentación

- Generar un ambiente de conferencia simulada

- Implementación de un sistema de evaluación

- Análisis de los resultados

- Propuestas de futuras acciones

El estudio se ha realizado con alumnos de $2^{\circ}$ de grado en una asignatura de contenidos químicos. No obstante, en la presente comunicación, se describe desde un enfoque más generalista para facilitar que la innovación docente pueda ser implementada en otras asignaturas. 


\section{Desarrollo de la innovación}

Alumnos. La acción propuesta se ha aplicado en la asignatura de Análisis Químico I del Grado en Ciencia y Tecnología de los Alimentos. Está impartida por la Unidad Docente Química Analítica en la Escuela Técnica Superior de Ingeniería Agronómica y del Medio Natural. Es una asignatura de segundo curso (cuatrimestre B) con dos subgrupos. Los alumnos que participaron en la actividad fueron 83 (curso 15-16) y 77 (curso 16-17), respectivamente, siendo el 93,3\% y 95,1 \% de los alumnos matriculados.

Herramientas informáticas. Se utilizó la plataforma docente de e-learning PoliformaT basada en el LMS (Learnig Management System) open source Sakai (https://sakaiproject.org/). El profesor prepara y pone a disposición de los alumnos el material necesario para el desarrollo de las actividades. Cada alumno accede al programa con su clave personalizada para descargarse los ficheros, colgar los informes y completar el cuestionario. Para el acceso de los alumnos a las fuentes bibliográficas, se han recomendado los portales genéricos Google Académico (https://scholar.google.es/), Scopus (https://www.scopus.com) y Polibuscador (http://polibuscador.upv.es); para apoyar la traducción de textos, se han recomendado los portales Google Traductor (https://translate.google.es/) y poli [Traductor] (https://politraductor.upv.es). La herramienta anti-plagio recomendada es Plagiarisma (http://plagiarisma.net/).

El análisis estadístico de las evidencias de la experiencia se realizó con Statgraphics Centurion XVI a un nivel de confianza del $95 \%$ (www.statgraphics.net).

Descripción de las actividades. La aproximación propuesta de aprendizaje por indagación ha sido aplicada para profundizar en temáticas especializadas de la asignatura. Cada grupo de 5-6 alumnos aborda un tema de interés en análisis químico para el sector agroalimentario, entre un listado que propone el profesor (menú Recursos de PoliformaT).

Existen una serie de tareas, denominadas pre-seminario, que comienzan seis semanas antes de la sesión de posters (menú Calendario de PoliformaT). Siguiendo un modelo de aprendizaje colaborativo, los alumnos en grupo buscan, analizan y procesan información relevante del campo en estudio. El progreso de estas actividades y del desarrollo del trabajo en equipo está supervisado por el profesor mediante tutorías a demanda o programadas. El documento resultante se envía a la plataforma de e-learning en una zona de espacio compartido (menú Espacio Compartido de PoliformaT) para que puedan acceder los distintos miembros del curso (profesor y el resto de compañeros) y aportar una retro-alimentación (menú Tareas de PoliformaT). También, permite evaluar las nuevas versiones y establecer si se han realizado las modificaciones sugeridas.

El seminario, denominado workshop en "Tendencias actuales en el análisis químico", se realiza en dos sesiones de 90 min cada una, en la clase y horario habitual de la asignatura. Para simular una reunión científico-técnica, se reorganiza el mobiliario del aula, los póster se cuelgan en las propias paredes del aula y en paneles y se generan pequeños espacios de discusión grupal. La primera parte del seminario es analizar los posters preparados por el resto de grupos, estableciendo una secuencia rotatoria con aproximadamente $5 \mathrm{~min} /$ póster. En la segunda parte, cada grupo de alumnos se reúne con el profesor para compartir los

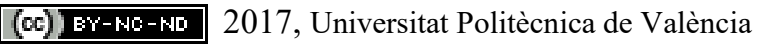


resultados de la primera observación. Se debate sobre los puntos fuertes y puntos débiles de los posters presentados, incidiendo especialmente en el preparado por el grupo (aprox. $5 \mathrm{~min}$ ). Durante ese tiempo el resto de alumnos completa una rúbrica de evaluación. En la tercera parte del seminario se realiza una breve exposición oral del póster por todos los miembros de un grupo $(10 \mathrm{~min}) \mathrm{y}$, posteriormente, se dedica un tiempo para que el resto de los grupos y el profesor realicen alguna pregunta $(5 \mathrm{~min})$. Tanto el profesor como los alumnos, rellenan una rúbrica sobre el desarrollo de la defensa. El seminario concluye con un análisis grupal de la experiencia, resaltando los objetivos de aprendizaje alcanzados. Además se completa la rúbrica, valorando globalmente la experiencia.

Se recogieron las evidencias de la experiencia desde el servidor de la UPV y se realizó el tratamiento de datos, el análisis de los resultados y las propuestas de mejora.

\section{Resultados}

\section{Desarrollo de las actividades previas a la sesión de posters}

La elaboración del póster se ha planificado como una actividad de aprendizaje por indagación en formato grupal. La experiencia ha mostrado que la clave del éxito de la actividad depende drásticamente de que los alumnos comprendan desde el primer momento los objetivos de aprendizaje que se buscan al realizarla. Particularmente, deben conocer las propiedades de un póster como herramientas de comunicación científico-técnica. También deben de disponer de directrices claras sobre la obtención de información que alimentará los contenidos del póster y el modo de plasmarlos en un reducido espacio, de forma clara y comprensible para un público con conocimientos suficientes pero no especialista en el campo que se recoge en el mismo. Aunque se incentive que cada grupo presente un póster con su propio estilo, debe asegurarse que el póster posea la suficiente calidad para que origine un aprendizaje significativo en los compañeros cuando sea expuesto y posteriormente defendido.

Nuestra innovación basada en el uso de herramientas como la plataforma de e-learning PoliformaT ha resultado ser una aproximación excelente para conseguir el objetivo marcado. La Figura 1 muestra la red de interconexiones diseñadas para la generación del póster. El nódulo central es el conjunto de dispositivos de cada grupo de alumnos. Éstos están conectados con la red global y con los servidores que controlan la plataforma. La figura recoge también el listado de tareas que pueden ser realizadas en cada uno de los nódulos. La hipótesis de trabajo planteada era que se gestionasen ciertas tareas clave desde la plataforma de e-learning. La funcionalidad asignada abarca desde facilitar los recursos necesarios (ej. plantillas, modelos) hasta regular el desarrollo (ej. plazos, retro-alimentación). 


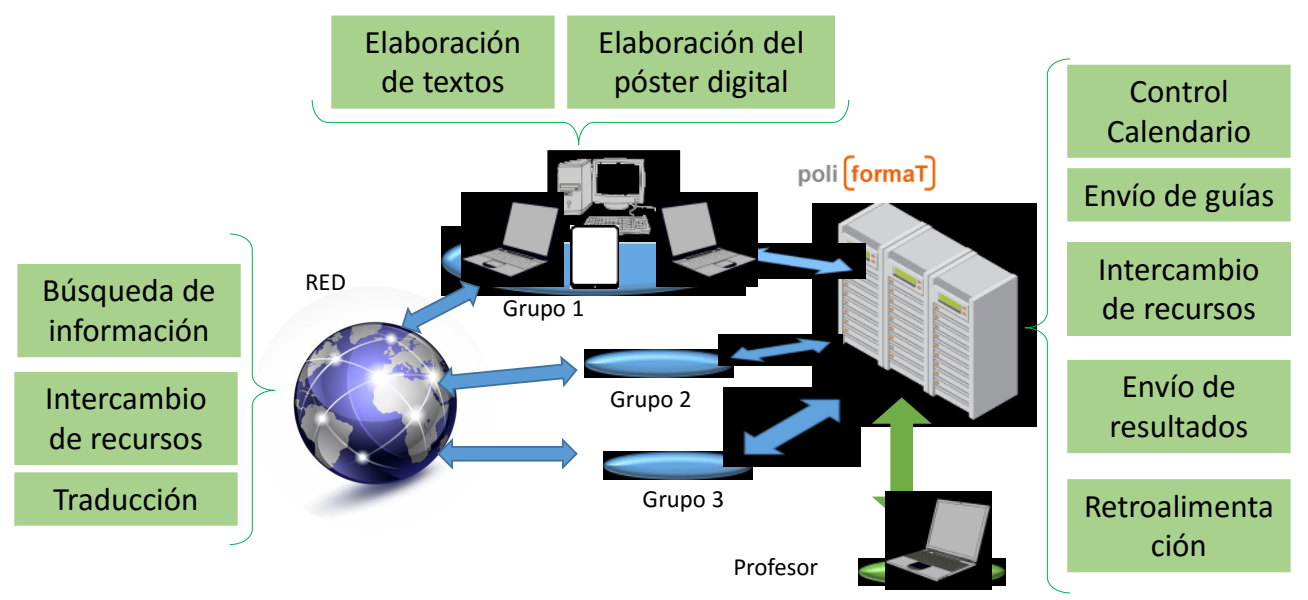

Fig. 1. Esquema de conectividad para la generación del póster. Los recuadros indican las actividades realizadas en cada nódulo.

Frente a aproximaciones anteriores basadas en papel o uso de plataformas digitales de forma puntual, las ventajas observadas al aplicar este enfoque son numerosas. La primera es que el alumno, a nivel individual y en grupo, están asistidos de forma accesible, disponiendo de los recursos elaborados por el profesor o por el resto de compañeros desde cualquier dispositivo. Los recursos preparados por el profesor actúan como guías, recogiendo ejemplos de actividades correctamente realizadas y de errores comúnmente cometidos por alumnos de anteriores promociones, facilitando el aprendizaje por indagación. Se suministran como material digital almacenado en un servidor educativo de acceso a los alumnos de la asignatura. Esto repercute en la capacidad de realizar la actividad en diferentes escenarios (casa particular, espacios de trabajo en el campus, etc) y facilita el trabajo en grupo incluso en el caso que los alumnos se encuentren físicamente distantes. La flexibilidad siempre repercute positivamente especialmente cuando se tratan de acciones grupales.

La segunda ventaja es que se obtienen evidencias del progreso de la actividad. Esto permite confirmar que los alumnos avanzan por el camino correcto y promueve un sentido de la responsabilidad personal y grupal que puede ir unido a una motivación por constatar la materialización de cada etapa del trabajo realizado. Además, se puede regular una adecuada gestión del tiempo, si se fijan plazos para los hitos intermedios del proyecto. En este sentido, para próximos cursos, se está analizando la implementación de registros o listas de verificación que faciliten la gestión de la actividad grupal y la consecución de los objetivos marcados de forma progresiva y no precipitada.

La tercera ventaja deriva de la capacidad para abrir vías para la retro-alimentación por la existencia de espacios compartidos en la plataforma y de menús específicos para cumplir con este fin. La consecuencia es una mejora la calidad del aprendizaje, evita confusiones o ambigüedades y ayudar a buscar soluciones alternativas más eficaces. Se trata de una evaluación formativa más que una simple calificación. Hay que indicar que este apoyo a través de la plataforma no es incompatible con otras vías de comunicación profesor-alumno (ej. correo electrónico, tutorías presenciales). En los cursos que se ha realizado la innovación, esta retro-alimentación sólo deriva de la evaluación por los distintos miembros del grupo y

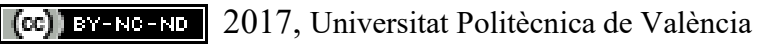


del profesor. No obstante, sería interesante explorar formas de implementar una retroalimentación basada en un tipo de evaluación constructivista por pares. A través de la plataforma PoliformaT, podría diseñarse una pequeña tarea con la finalidad de comparar los trabajos de los compañeros con la propia labor realizada. De este modo, los aspectos positivos y negativos se transmitirían de forma inter-grupal y se contribuiría a una revisión del proceso y no únicamente del producto final.

\section{Sesión de posters}

Las tareas grupales culminan en un seminario especializado (workshop) que simula las condiciones de una reunión científica o seminario comercial. Los alumnos presentan en formato cartel los posters que han sido revisados a través de la plataforma PoliformaT (Figura 2). Cualquier programa para la creación del póster digital es válido (Microsoft PowerPoint, GoogleSlides, KeyNote), pero se restringió el envío en formato pdf para garantizar la compatibilidad entre dispositivos. Todos los posters deben incluir: Título, Nombre de los alumnos, Filiación, Descripción de la técnica, Ejemplo práctico, Tendencias de futuroConclusión, Referencias. La composición y estructura del póster es libre, aunque se fija un rango de dimensiones.

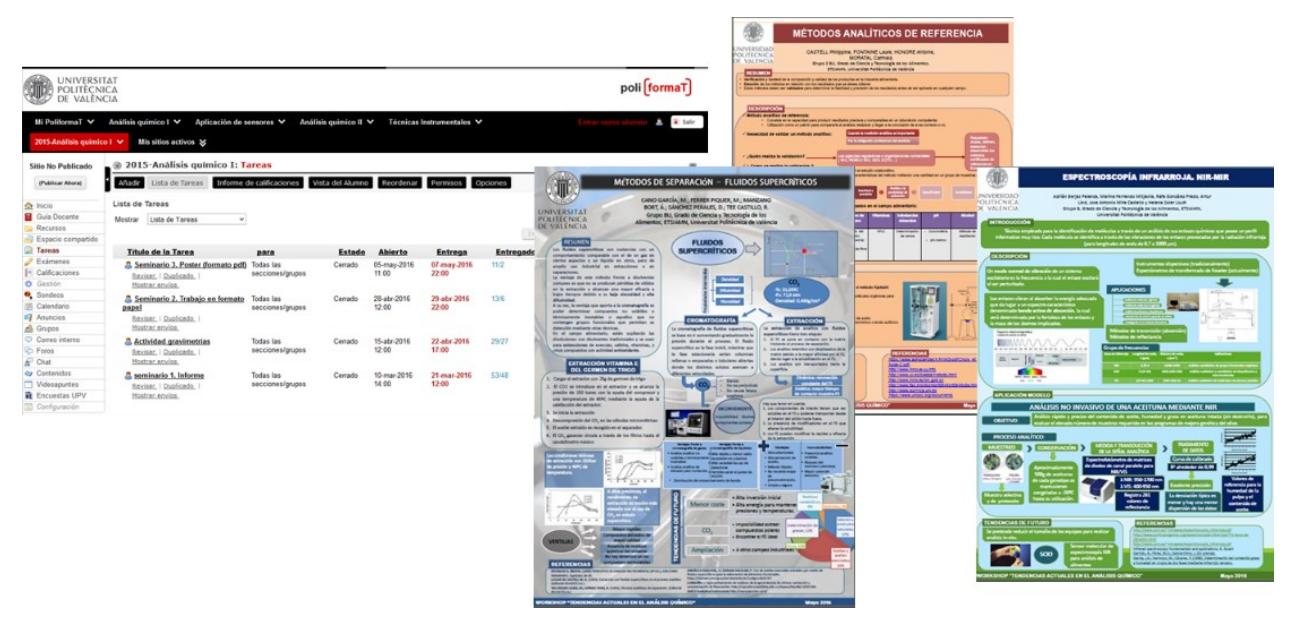

Fig. 2. Captura de pantalla y posters elaborados por los alumnos en formato digital compartidos a través de la plataforma e-learning

En los espacios habilitados en el aula y en los entornos de los posters, los alumnos discutieron sobre el trabajo realizado por los compañeros, comparándolo con el realizado por uno mismo. Así, se logra un escenario educativo donde desarrollaran sus habilidades de comunicación oral. La conclusión del ejercicio es una presentación oral colaborativa sobre el contenido del póster al conjunto de la clase. Se consiguió que la defensa fuese ágil, concisa y enfocada a transmitir las características fundamentales de la técnica o tema de estudio y sus aplicaciones, evitando una mera lectura del póster. El profesor actúa de moderador y ejerce un papel más activo solamente para solucionar aquellas dudas que no pueden ser contestadas por el grupo. 
Se confirmó la hipótesis del presente estudio, es decir, el éxito de esta sesión depende del trabajo realizado con anterioridad. La organización y planificación previa del profesor tiene que ser suficiente para que los alumnos adopten una posición protagonista y sean el auténtico motor de la actividad. Para ello, debe haber suministrado suficiente información que permita ese elevado grado de independencia sin perder de vista que se trata de una actividad académica con unos claros objetivos de aprendizaje. Del mismo modo se comprobó que este tipo de actividades son fundamentales para superar las principales carencias constatadas en profesionales universitarios, tales como habilidades relacionadas con la comunicación, pensamiento crítico y trabajo en grupo (Asharf et al. 2010).

\section{Evaluación}

El desarrollo de la experiencia es valorada por los alumnos mediante rúbricas (evaluación por iguales) y por el profesor. La Tabla la recoge los aspectos del póster que fueron evaluados. Se centran principalmente sobre los objetivos de aprendizaje relacionados con la temática en estudio y sobre los aspectos más formales o de diseño. El alumno al participar del proceso de evaluación afianza que atributos del póster son prioritarios y cuales son secundarios, comprendiendo que su finalidad es proporcionar información sintetizada pero comprensible y útil. Su finalidad es el desarrollo del pensamiento crítico, vía observación guiada, resaltando los efectos positivos del aprendizaje cuando se realiza de manera reflexiva.

Tabla 1. Criterios de valoración del póster (a) y de la exposición (b)

(a)

\begin{tabular}{ll}
\hline Criterio & Comentario \\
\hline Contenido & $\begin{array}{l}\text { La información presentada es relevante, específica y exacta, abordando el } \\
\text { fundamento, descripción y aplicaciones de la técnica. El ejemplo de aplicación } \\
\text { ayuda a comprender la técnica. } \\
\text { Las distintas secciones del póster están ordenadas y con la información } \\
\text { relevante claramente organizada y resaltada. } \\
\text { El lenguaje científico general y el vocabulario propio del área profesional son } \\
\text { conguaje }\end{array} \quad \begin{array}{l}\text { Diseño atractivo con toda la información legible y figuras de calidad. } \\
\text { Formato }\end{array}$ \\
Comprensión & importantes de la técnica y podría explicársela a un/una compañero/a. \\
\hline
\end{tabular}

(b)

\begin{tabular}{ll}
\hline Criterio & Comentario \\
\hline Lenguaje & $\begin{array}{l}\text { El lenguaje científico en general y el vocabulario propio del área profesional } \\
\text { son correctamente utilizados. }\end{array}$ \\
Liscurso & $\begin{array}{l}\text { La exposición es clara y organizada, centrándose en los aspectos relevantes de } \\
\text { la técnica }\end{array}$ \\
Resolución de las & Las distintas preguntas han sido contestadas de forma correcta y resolviendo \\
cuestiones & las dudas planteadas. \\
Comprensión & La información presentada ha permitido comprender los aspectos más \\
& importantes de la técnica y podría explicársela a un/una compañero/a.
\end{tabular}

La Tabla $1 \mathrm{~b}$ muestra los aspectos de la defensa grupal del póster evaluados en la rúbrica con su descripción. Las máximas calificaciones se alcanzan cuando el grupo expone su póster

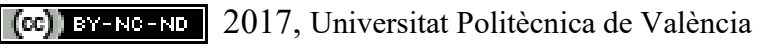


utilizando las características de la comunicación científica, y consigue transmitir los elementos objeto del aprendizaje. Los distintos grupos reciben una retro-alimentación de sus compañeros y del profesor en la propia sesión, de modo que se origina un escenario donde se profundiza en el aprendizaje sin generar una situación intimidatoria en el aula.

\section{Valoración de la experiencia docente}

La opinión de la experiencia innovadora por los estudiantes es recogida mediante una cuestión con respuesta en escala Likert. La pregunta era "Evalúa la aportación de la actividad del workshop (trabajo no presencial + presencial) en tu aprendizaje frente otras estrategias educativas más convencionales. 0: Nula, 1: Poca, 2: Normal, 3: Notable, 4: Destacada”. La Figura 3 recoge la frecuencia de cada una de las respuestas para los datos recogidos en el primer curso. La mayoría de los alumnos (97 \%) otorgaron una valoración positiva del impacto de la actividad sobre su aprendizaje. Estos resultados coinciden con las observaciones recogidas durante el propio desarrollo y con las manifestaciones indicadas directamente a los profesores. Los principales comentarios positivos son el orgullo sobre el trabajo realizado y la novedad para abordar un trabajo académico grupal. No obstante, el primer año de implementación de la experiencia también manifestaron la necesidad de mayor guía en la fase de pre-seminario, es decir, en la búsqueda de información y elaboración del póster. Por ello, la acción docente del segundo año se está centrando en mejorar dicho aspecto.

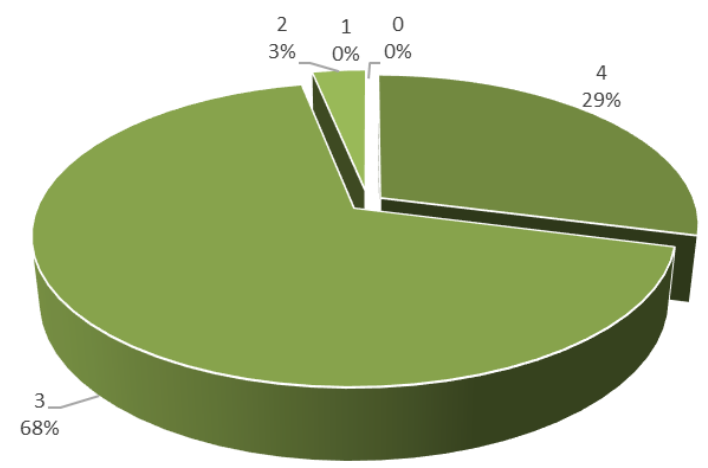

Fig. 3. Valoración global de la experiencia por los alumnos. 0: Nula, 1: Poca, 2: Normal, 3: Notable, 4: Destacada

\section{Conclusiones}

Las directrices de educación universitaria están enfocadas a promover actividades donde los estudiantes puedan tener una participación más activa. Nuestra experiencia ha confirmado la máxima en educación que dice que una tarea abordada en profundidad origina un aprendizaje más significativo y relevante que la realización de una multitud de pruebas superficiales. Nuestra propuesta de aprendizaje por indagación combinada con una sesión de posters, es una aproximación que permite fomentar ciertas habilidades imprescindibles pero 
generalmente no instruidas en modelos tradicionales de enseñanza. Este objetivo se logra sin renunciar a contenidos básicos de una titulación universitaria.

Su aplicación en una asignatura con contenidos químicos ha sido satisfactoria, dando lugar a una actividad práctica centrada en el problema, que estimula la relación del conocimiento con una cuestión específica de interés y favorece la comprensión. En este entorno de aprendizaje, los alumnos se aproximan a la realidad de la investigación científica actual. Al apoyarse en una plataforma de e-learning, es más fácil la adaptación a la pluralidad escenarios que se pueden originar en un trabajo en grupo (ej. incompatibilidades de horarios para reunirse). La presentación en una sesión de posters es una estrategia que conduce a un ambiente agradable y gratificante para los estudiantes. En este escenario, se logra que expongan con naturalidad comentarios sobre sus trabajos y el de los compañeros de una manera constructiva, reflexionen sobre los errores cometidos y puedan encontrar puntos de apoyo para próximas situaciones.

En futuras aplicaciones de la estrategia docente, se prevén incluir acciones que podría ayudar a complementar la experiencia. Primero, se puede revisar el proceso de retroalimentación de los trabajos/posters subidos a la plataforma. Una opción a estudiar es considerar la asignación aleatorizada de cada trabajo para una revisión por pares. Posteriormente, cada grupo argumente los cambios que ha realizado en base a las sugerencias de sus revisores previo a la presentación definitiva en el seminario. Segundo, las rúbricas de evaluación se han realizado en formato papel, mientras que en próximos cursos, se plantea el uso de rúbricas digitales (menú Exámenes de PoliformaT). Tercero, las actividades sugeridas, con el formato actual, no presentan un claro control del aprendizaje colaborativo. Una futura aproximación es la incorporación de dinámicas de aprendizaje cooperativo, estableciendo roles y mecanismos de control del aprendizaje por los propios alumnos. Cuarto, la utilización por el alumnado de recursos web colaborativos, como https://awwapp.com/, https://realtimeboard.com/o https://ziteboard.com/, podría ayudar en la elaboración digital del póster.

Aunque existe todavía un margen importante de mejora, se trata de una actividad recomendable para ser transferida a otras asignaturas.

\section{Referencias}

ASHRAF, S. S., MARZOUK, S. A., SHEHADI, I. A., \& MURPHY, B. M. (2010). “An integrated professional and transferable skills course for undergraduate chemistry students". Journal of Chemical Education, 88(1), 44-48.

CARABANTES, D. (2010). "Innovación en la creación de materiales y actividades para la enseñanza en red”. Relada 4,4, pp. 290-297.

DENZINE, G. M. (1999). “An example of innovative teaching: Preparing graduate students for poster presentations". Journal of College Student Development, 40(1), 91-93.

EDELSON, D. C., GORDIN, D. N., \& PEA, R. D. (1999). "Addressing the challenges of inquiry-based learning through technology and curriculum design". Journal of the Learning Sciences, 8(3-4), 391-450.

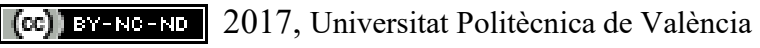


GORMALLY, C., BRICKMAN, P., HALLAR, B., \& ARMSTRONG, N. (2009). "Effects of inquiry-based learning on students' science literacy skills and confidence". International Journal for the Scholarship of Teaching and Learning, 3(2), 16.

KAARTINEN, S., \& KUMPULAINEN, K. (2002). "Collaborative inquiry and the construction of explanations in the learning of science". Learning and Instruction, 12(2), 189-212.

MARINO, R., CLARKSON, S., MILLS, P. A., SWEENEY, W. V., \& DEMEO, S. (2000). "Using poster sessions as an alternative to written examination-the poster exam". Journal of Chemical Education, 77(9), 1158.

NEWBREY, M. AND BALTEZORE, J. (2006). "Poster Presentations: Conceptualizing, Constructing and Critiquing". American Biology Teacher 68(9), 550-4.

REDONDO, F. (2008). "Trabajo colaborativo, espacio digital y e-learning”. Relada 2,3, pp.143-149.

ROFFE, I. (2002). "E-learning: engagement, enhancement and execution", Quality Assurance in Education, 10, 40-50.

SCOTT, P. (2008). "Poster Presentations: An Effective Assessment Tool". Journal of the Further Education Alliance, 2, 5-16.

SISAK, M. (1997). "Poster Sessions as a Learning Technique". Journal of Chemical Education, 74(9), 1065-1067.

STEGEMANN, N., \& SUTTON-BRADY, C. (2009). "Poster sessions in marketing education an empirical examination". Journal of Marketing Education, 31(3), 219-229.

VAN JOOLINGEN, W. R., DE JONG, T., \& DIMITRAKOPOULOU, A. (2007). "Issues in computer supported inquiry learning in science". Journal of Computer Assisted Learning, 23(2), 111-119.

WIMPFHEIMER, T. (2004). "Peer-evaluated poster sessions: an alternative method to grading general chemistry laboratory work". Journal of Chemical Education, 81(12), 1775.

WENZEL, T. J. (2007). "Collaborative and project-based learning in analytical chemistry" en Active Learning, ACS Symposium Series, Chapter 5, pp 54-68

YANG, Y. F. y TSAI, C. C. (2010). "Conceptions and approaches to learning through online peer assessment”, Learning and Instruction, 20, 72-83. 